\title{
Sound Event Detection and Time-Frequency Segmentation from Weakly Labelled Data
}

\author{
Qiuqiang Kong*, Yong Xu*, Iwona Sobieraj, Wenwu Wang, Mark D. Plumbley Fellow, IEEE
}

\begin{abstract}
Sound event detection (SED) aims to detect when and recognize what sound events happen in an audio clip. Many supervised SED algorithms rely on strongly labelled data which contains the onset and offset annotations of sound events. However, many audio tagging datasets are weakly labelled, that is, only the presence of the sound events is known, without knowing their onset and offset annotations. In this paper, we propose a time-frequency (T-F) segmentation framework trained on weakly labelled data to tackle the sound event detection and separation problem. In training, a segmentation mapping is applied on a T-F representation, such as log mel spectrogram of an audio clip to obtain T-F segmentation masks of sound events. The T-F segmentation masks can be used for separating the sound events from the background scenes in the time-frequency domain. Then a classification mapping is applied on the T-F segmentation masks to estimate the presence probabilities of the sound events. We model the segmentation mapping using a convolutional neural network and the classification mapping using a global weighted rank pooling (GWRP). In SED, predicted onset and offset times can be obtained from the T-F segmentation masks. As a byproduct, separated waveforms of sound events can be obtained from the T-F segmentation masks. We remixed the DCASE 2018 Task 1 acoustic scene data with the DCASE 2018 Task 2 sound events data. When mixing under $0 \mathrm{~dB}$, the proposed method achieved F1 scores of $0.534,0.398$ and 0.167 in audio tagging, frame-wise SED and event-wise SED, outperforming the fully connected deep neural network baseline of $0.331,0.237$ and 0.120 , respectively. In T-F segmentation, we achieved an F1 score of 0.218 , where previous methods were not able to do T-F segmentation.
\end{abstract}

Index Terms-Sound event detection, time-frequency segmentation, weakly labelled data, convolutional neural network.

\section{INTRODUCTION}

Sound event detection (SED) aims to detect what sound events happen in an audio recording and when they occur. SED has many applications in everyday life. For example, SED can be used to monitor "baby cry" sound at home [1], and to detect "typing keyboard", "door slamming", "ringing of phones", "smoke alarms" and "sirens" in the office $[2,3]$. For public security, SED can be used to detect "gunshot" and "scream" sounds [4]. Not only is SED complementary to video or image based event detection [5]-[7] but also has many advantages over the two modalities. First, sound does not require illumination, so can be used in dark environments. Second, sound can penetrate or move around some obstacles, while objects in video and image are often occluded. Third, some abnormal events such as fire alarms are audio only, so can only be detected by sound. Furthermore, storing and processing sound often consumes less computation resources

* The first two authors contributed equally to this work.
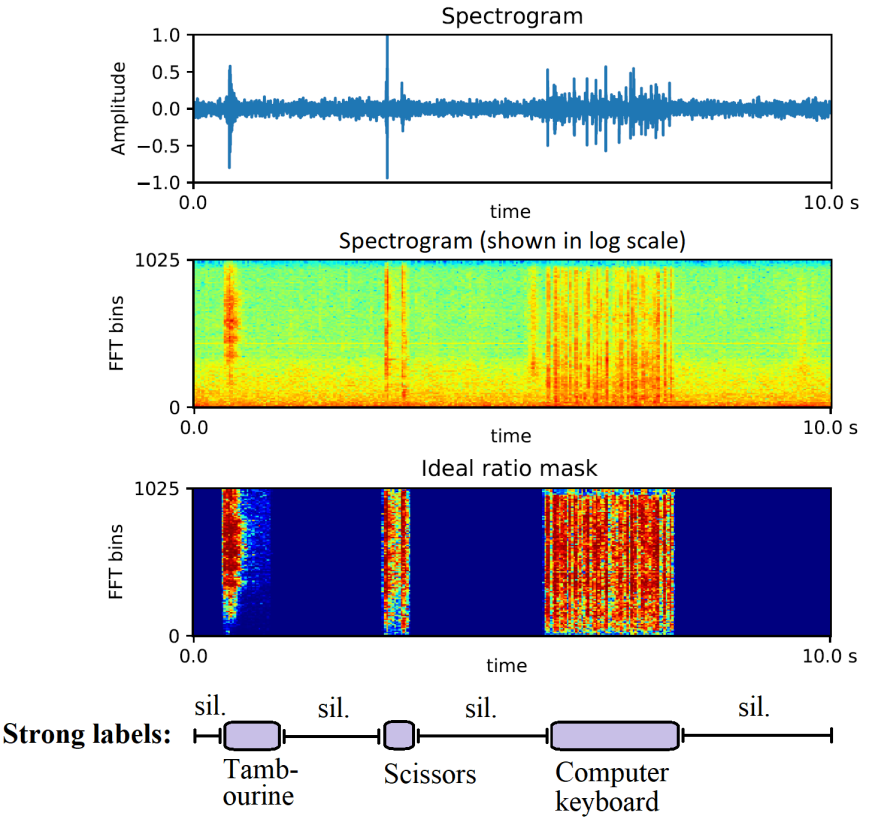

Weak labels: Scissors, computer keyboard, tambourine

Fig. 1. From top to bottom: Waveform of an audio clip containing three sound events: "Tambourine", "scissors" and "computer keyboard"; Log mel spectrogram of the audio clip; Ideal ratio mask (IRM) [9] of sound events. Strongly labelled onset and offset annotations of sound events; Weak labels. "Silence" is the abbreviated as "sil.". The signal-to-noise ratio of this audio clip is $0 \mathrm{~dB}$.

than video [8], and as a result, longer sound sequences can be stored in a device and faster processing can be obtained using equal computation resources. Many SED algorithms rely on strongly labelled data [10]-[12] where the onset and offset times of sound events have been annotated. The segments between the onset and offset labels are used as target events for training, while those outside the onset and offset annotations are used as non-target events [11, 12]. However, collecting strongly labelled data is time consuming because annotating the onset and offset times of sound events takes more time than annotating audio clips for classification, so the sizes of strongly labelled datasets are often limited to minutes or a few hours $[12,13]$. At the same time there are large amounts of weakly labelled data (WLD) available, where only the presence of the sound events is labelled, without any onset and offset annotations [14, 15] or the sequence of the sound events. Fig. 1 shows the waveform of an audio clip containing three non-overlapping sound events, the log mel spectrogram of the audio clip, the ideal ratio mask (IRM) [9] of the sound 


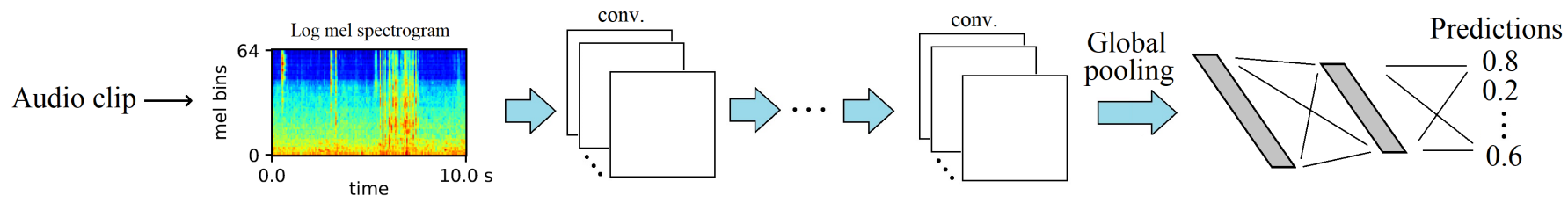

Fig. 2. Audio tagging with convolutional neural network. Input log mel spectrogram is presented to a convolutional neural network including convolutional layers, a global pooling layer and fully connected layers to predict the presence probabilities of audio tags.

events, the strongly labelled onset and offset annotations and the weak labels. In this paper we will focus on non-overlapping sound events as a starting point.

In the real world, sound events usually happen in real scenes such as a metro station or an urban park. State-of-the-art SED algorithms only detect the onset and the offset of sound events in the time domain but do not separate them from background in the T-F domain. The separation of sound events in the T-F domain can be useful for enhancing and recognizing sound events in audio scenes under low signal-to-noise ratio (SNR). In this paper, we propose a T-F segmentation and sound event detection framework trained using weakly labelled data. This is done by learning $T-F$ segmentation masks implicitly in training with only the clip-level audio tags. It means that T-F masks are not known even for the training set: they are predicted as intermediate results. T-F segmentation masks are equivalent to the ideal ratio masks (IRM) [9]. An IRM is the ratio of the spectrogram of a sound event to the spectrogram of the mixed audio. T-F segmentation masks can be used for SED and sound event separation. In training, a segmentation mapping is applied to the T-F representation such as log mel spectrogram of an audio clip to obtain T-F segmentation masks for sound events. Then a classification mapping is applied to the T-F segmentation masks to output the presence probabilities of sound events. In T-F segmentation, with a T-F representation of an audio clip as input, the trained segmentation mapping is used to obtain the T-F segmentation masks. In SED, onset and offset times can be obtained from the T-F segmentation masks. As a byproduct, separated waveforms of sound events can be obtained from the T-F segmentation masks. This work is an extension of the joint separation-classification model for SED of weakly labelled data [16].

The paper is organized as follows. Section II introduces previous work in SED with WLD. Section III describes the proposed T-F segmentation, sound event detection and separation framework. Section IV describes the implementation details of the proposed framework. Section V shows experimental results. Section VI concludes and forecasts future work.

\section{WEAKLY SUPERVISED SOUND EVENT DETECTION}

Compared to the conventional SED task, where strongly labelled onset and offset annotations for the training set are given, the weakly supervised SED task contains only clip-level labels. That is, only the presence of sound events is known in an audio clip, without knowing the temporal locations of the events. Several approaches for weakly supervised SED have recently been proposed, including multiple instance learning and convolutional neural networks.

\section{A. Multi-instance learning method}

One solution to the WLD problem is based on multiple instance learning (MIL) [14, 17]. MIL was first proposed in 1997 for drug activity detection [18]. In MIL for SED, an audio clip is labelled positive for a specified sound event if that sound event occurs at least one time in the audio clip, and labelled negative if that sound event does not occur in the audio clip. For strongly labelled data, the dataset consists of training pairs $\{x, y\}$ where $x$ is the feature of a frame in an audio clip and $y \in\{0,1\}^{K}$ is the strong label of the frame, where $K$ denotes the number of sound classes. For weakly labelled data, features of all frames in an audio clip constitute a bag $B=\left\{x_{t}\right\}_{t=1}^{T}$ where $T$ is the number of frames in the audio clip. Multiple instance assumption states that the weak labels of a bag are $y=\max _{t}\left\{y_{t}\right\}_{t=1}^{T}$, where $y_{t}$ is the strong label of the feature $x_{t}$. The weakly labelled data consists of the training pairs $\{B, y\}$.

The problem of SED from WLD now can be cast as learning a classifier to predict the labels of the frames $\left\{y_{t}\right\}_{t=1}^{T}$ of a bag $B=\left\{x_{t}\right\}_{t=1}^{T}$. For the general WLD problem, an MIL framework based on a neural network was proposed in $[14,19]$. In $[14,20]$ a support vector machine (SVM) was used to solve MIL as a maximum margin problem. A negative mining method was proposed in [21] that selects negative examples according to intra-class variance criterion. A concept ranking according to negative exemplars (CRANE) algorithm was proposed in [22]. However, an MIL method tends to underestimate the number of positive instances in an audio clip [23]. Furthermore, the MIL method cannot predict the T-F segmentations from the WLD [14].

\section{B. Convolutional neural networks for audio tagging and weakly supervised sound event detection}

Convolutional neural networks (CNNs) have been successfully used in many areas including image classification [24], object detection [6], image segmentation [25], speech recognition [26, 27] and audio classification [28]. In this section we briefly introduce previous work using convolutional neural network for audio tagging [28] and weakly supervised SED.

Audio tagging $[12,28,29]$ aims to predict the presence of sound events in an audio clip. In [30], a mel spectrogram of an audio clip is presented to a CNN, where the filters of each convolutional layer capture local patterns of a spectrogram. After a global pooling layer such as global max pooling [28], global 


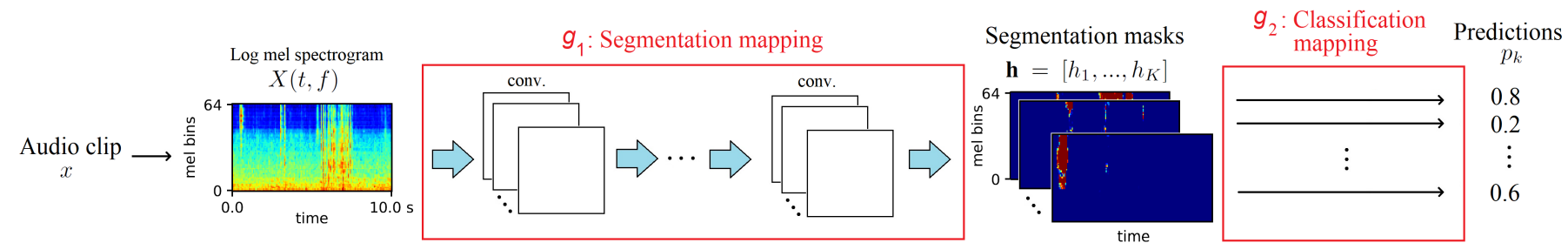

Fig. 3. Training stage using weakly labelled data. A segmentation mapping $g_{1}$ maps from an input T-F representation to the segmentation masks. A classification mapping $g_{2}$ maps each segmentation mask to the presence probabilities of the corresponding audio tag.

average pooling [31], global weighted rank pooling [23], global attention pooling [32, 33] or other poolings [34, 35], fully connected layers are applied to predict the presence probabilities of audio classes. Fig. 2 shows the framework of audio tagging with convolutional neural network. However, this CNN only predicts the presence probabilities of a sound events in an audio clip, but not the onset and offset times of the sound events.

In $[36,37]$, a time-distributed $\mathrm{CNN}$ with a global maxpooling strategy was proposed to approximate the MIL method to predict the temporal locations of each event. However, the global max-pooling will encourage the model to attend to the most dominant T-F unit contributing to the presence of the sound event and ignore all of other T-F units. That is, the happening time of the sound events is underestimated. A method for localizing the sound events in an audio clip by splitting the input into several segments based on the CNNs was presented in [38]. It splits an audio clip into several segments with the assumption that parts of the segments correspond to the cliplevel labels. This assumption may be unreasonable due to the fact that some sound events may only occur at certain frames. Recently, an attention-based global pooling strategy using CNNs was proposed to predict the temporal locations [39] for SED using WLD. However, attention-based global pooling can only predict the time domain segmentation, but not the T-F segmentation which will be firstly addressed in this paper.

\section{TIME-FREQUENCY SEGMENTATION, SOUND EVENT DETECTION AND SEPARATION FROM WEAKLY LABELLED DATA}

In this section, we present a T-F segmentation, sound event detection and separation framework trained on weakly labelled audio data. Unlike the CNN method for audio tagging, we design a CNN to learn T-F segmentation masks of sound events from the weakly labelled data.

\section{A. Training from weakly labelled data}

We use only weakly labelled audio data to train the proposed model. The training stage is shown in Fig. 3. To begin with, the waveform of an audio clip $x$ is converted to an input timefrequency (T-F) representation $X(t, f)$, for example, spectrogram or log mel spectrogram. To simplify the notation, we abbreviate $X(t, f)$ as $X$.

The first part of the training stage is a segmentation mapping $g_{1}: X \mapsto \mathbf{h}$ which maps the input T-F representation to the T-F segmentation masks $\mathbf{h}=\left[h_{1}, \ldots, h_{K}\right]$, where $K$ is the number of T-F segmentation masks and is equal to the number of sound events. Symbol $h_{k}$ is the abbreviation of $h_{k}(t, f)$ which is the T-F segmentation mask of the $k$-th event. Ideally, each T-F segmentation mask $h_{k}$ is an ideal ratio mask [9] of the $k$-th sound event.

The second part of the training stage is a classification mapping $g_{2}: h_{k} \mapsto p_{k}, k=1, \ldots, K$ where $g_{2}$ maps each T-F segmentation mask to the presence probability of the $k$-th event, denoted as $p_{k}$. Then the binary crossentropy between the predictions $p_{k}, k=1, \ldots, K$ and the targets $y_{k}, k=1, \ldots, K$ is calculated as the loss function:

$$
\begin{aligned}
l\left(p_{k}, y_{k}\right) & =-\sum_{k=1}^{K} y_{k} \log p_{k} \\
& =-\sum_{k=1}^{K} y_{k} \log g_{2}\left(g_{1}(X)_{k}\right),
\end{aligned}
$$

where $y_{k} \in\{0,1\}, k=1, \ldots, K$ is the binary representation of the weak labels. Both $g_{1}$ and $g_{2}$ can be modeled by neural networks. The parameters of $g_{1}$ and $g_{2}$ can be trained end-toend from the input T-F representation to the weak labels of an audio clip.

\section{B. Time-frequency segmentation}

In inference step, the input T-F representation of an audio clip is presented to the segmentation mapping $g_{1}$ to obtain the T-F segmentation masks $h_{k}, k=1, \ldots, K$. The T-F segmentation masks indicate which T-F units in the T-F representation contribute to the presence of the sound events (top right of Fig. 4). The learned T-F segmentation masks are affected by the classification mapping $g_{2}$ and will be discussed in Section IV.

\section{Sound event detection}

As T-F segmentation masks $h_{k}, k=1, \ldots, K$ contain the information about where sound events happen in the T-F domain, the simplest way to obtain the sound event detection score $v_{k}(t)$ in the time domain is to average out the frequency axis of the T-F segmentation masks (bottom right of Fig. 4):

$$
v_{k}(t)=\frac{1}{F} \sum_{f=1}^{F} h_{k}(t, f),
$$

where $F$ is the number of frequency bins of the segmentation mask $h_{k}$. Then $v_{k}(t)$ is the score of the frame-wise prediction 

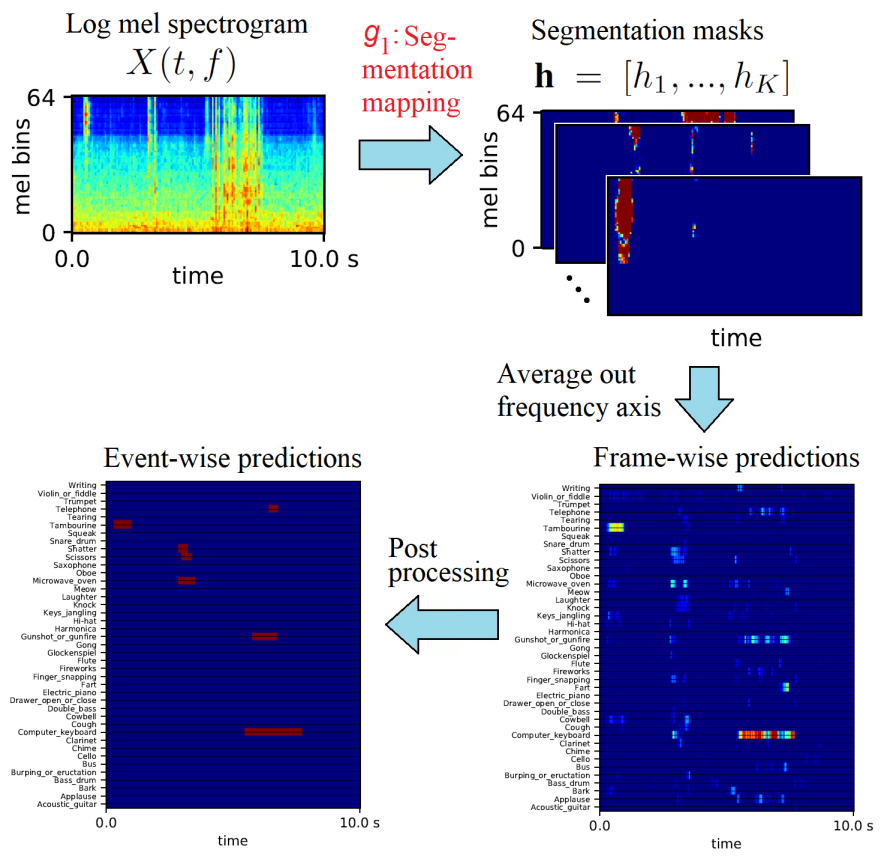

Fig. 4. Inference stage. An input T-F representation is presented to the segmentation mapping $g_{1}$ to obtain the T-F segmentation masks. By averaging out the frequency axis of the T-F segmentation masks and post processing, event-wise predictions of sound events can be obtained.

of the sound events. We describe how to convert the framewise scores to event-wise sound events in Section IV-C.

\section{Sound event separation}

As a byproduct, the T-F segmentation masks can be used to separate sound events from the mixture in the T-F domain. In addition, by applying an inverse Fourier transform on the separated T-F representation of each sound event, separated waveforms of the sound events can be obtained. Separating sound events from the mixture of sound events and background under a low SNR can improve the recognition of sound events in future work. Fig. 5 shows the pipeline of sound event separation. An audio clip $x$ is presented to the segmentation mapping $g_{1}$ to obtain T-F segmentation masks. Meanwhile, the complex spectrum $\widetilde{X}$ of the audio clip is calculated. We use the tilde on $X$ to distinguish the complex spectrum $\widetilde{X}$ from the input T-F representation $X$ because $X$ might not be a spectrum, such as log mel spectrogram. We interpolate the segmentation masks of the input T-F representation $h_{k}, k=1, \ldots, K$ to $\widetilde{h}_{k}, k=1, \ldots, K$ representing the $\mathrm{T}-\mathrm{F}$ segmentation masks of the complex spectrum. The reason for performing this interpolation is that $\widetilde{h}_{k}$ may have a size different from $h_{k}$, for example, a log mel spectrogram has fewer frequency bins than linear spectrum in the frequency domain. Then we multiply the upsampled T-F segmentation masks $\widetilde{h}_{k}$ with the magnitude of the spectrum to obtain the segmented spectrogram of the $k$-th event:

$$
\widetilde{Y}_{k}=\widetilde{h}_{k} \odot|\tilde{X}|, k=1, \ldots, K,
$$

where $\odot$ represents the element-wise multiplication and $\widetilde{Y}_{k}$ represents the segmented spectrogram of the $k$-th event. Fi- nally, an inverse Fourier transform with overlap add [40] is applied on each segmented spectrogram with the phase from $\widetilde{X}$ to obtain the separated waveforms $\widehat{s}_{k}, k=1, \ldots, K$ :

$$
\widehat{s}_{k}=\operatorname{IFFT}\left(\widetilde{Y}_{k} \cdot e^{j \angle \tilde{X}}\right) .
$$

We summarize the training, time-frequency segmentation, sound event detection and separation framework in Fig. 6. The training stage, sound event detection stage and sound event separation stage are shown in the left, middle and right column of Fig. 6, respectively.

\section{PRoposed SEgMENTATION MAPPING AND CLASSIFICATION MAPPING}

In this section, we describe the implementation details of the segmentation mapping $g_{1}$ and the classification mapping $g_{2}$ proposed in Section III.

\section{A. Segmentation mapping}

Segmentation mapping $g_{1}$ takes a T-F representation of an audio clip as input and outputs segmentation masks of each sound event. We use log mel spectrogram as the input T$\mathrm{F}$ representation, which has been shown to perform well in audio classification $[28,39,41]$. Ideally, the outputs of $g_{1}$ are ideal ratio masks (IRMs) [42] of sound events in the T-F domain. The segmentation mapping $g_{1}$ is modeled by a CNN. Each convolutional layer consists of a linear convolution, a batch normalization (BN) [43] and a ReLU [44] nonlinearity as in [43]. The BN inserted between the convolution and the nonlinearity can stabilize and speed up the training [43]. We do not apply downsampling layers after convolutional layers because we want to retain the resolution of the input T-F segmentation masks. The T-F segmentation masks are obtained from the activations of the last CNN layer using a sigmoid nonlinearity to constrain the values of the T-F segmentation masks to be between 0 and 1 to be a valid value of an IRM. The configuration details of the CNN will be described in Section V-D.

The idea of learning the T-F segmentation masks explicitly is inspired by work on weakly labelled image localization [45] and image segmentation [46, 47]. In weakly labelled image localization, saliency maps are learned indicating the locations of the objects in an image [45]. Similarly, the T-F segmentation masks in our work resemble the saliency maps of an image [45], where T-F segmentation masks indicate what time and frequency a sound event occurs in a T-F representation.

\section{B. Classification mapping}

As described in Section III, the classification mapping $g_{1}$ maps each segmentation mask $h_{k}$ to the presence probability of its corresponding sound event. Modeling the classification mapping in different ways will lead to different representation of the segmentation masks (Fig. 7). We explored global max pooling [28], global average pooling [31] and global rank pooling [23] for modeling the classification mappings $g_{2}$. 


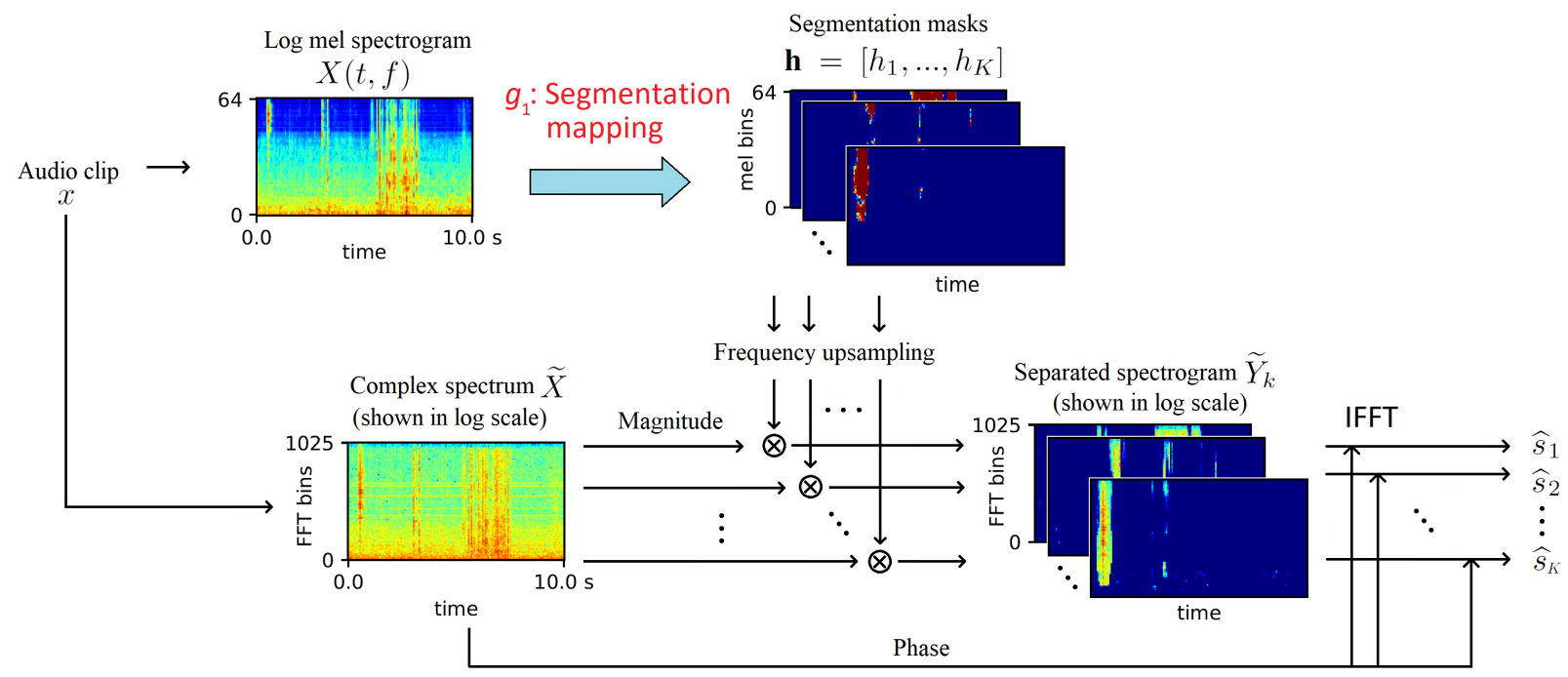

Fig. 5. Sound event separation stage. An input T-F representation is presented to the segmentation mapping $g_{1}$ to obtain the T-F segmentation masks. The upsampled segmentation masks are multiplied with the magnitude spectrum of the input audio to obtain the segmented spectrogram of each sound event. Separated sound events are obtained by applying an inverse Fourier transform to the segmented spectrogram.

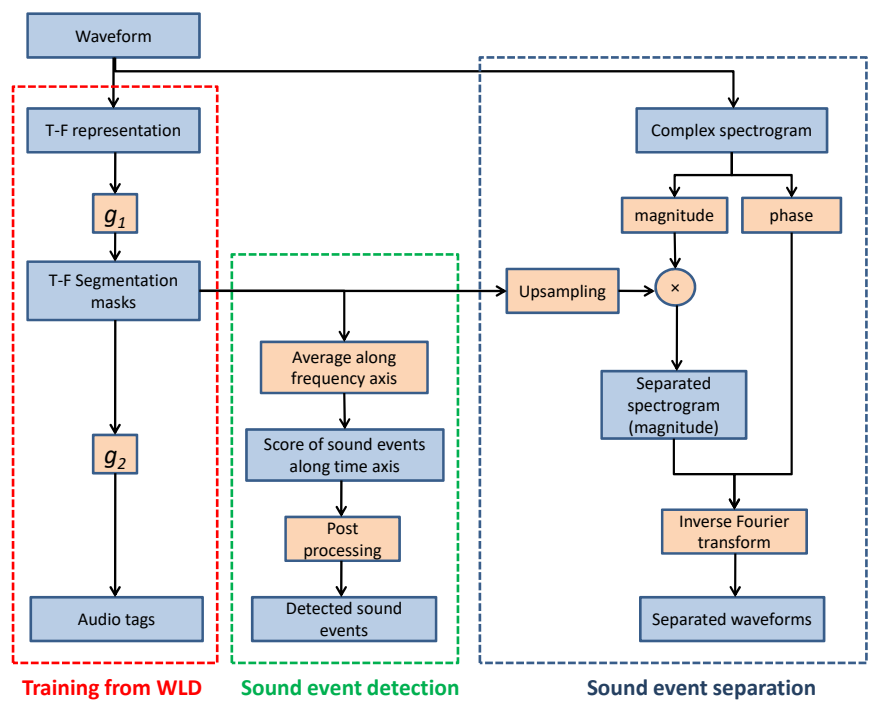

Fig. 6. Framework of T-F segmentation, sound event detection and sound event separation from WLD. From left to right: Training from WLD; Sound event detection; Sound event separation.

1) Global max pooling: Global max pooling (GMP) applied on feature maps has been used in audio tagging [28]. GMP on each T-F segmentation mask map $h_{k}$ is depicted as:

$$
F\left(h_{k}\right)=\max _{t, f} h_{k}(t, f) .
$$

GMP is based on the assumption that an audio clip contains a sound event if at least one T-F unit of the T-F input representation contains a sound event. GMP is invariant to the location of sound event in the T-F domain because whenever a sound event occurs, GMP will only select the maximum value of a T-F segmentation mask which is robust to the time or frequency shifts of the sound event. However, in the training stage, back propagation will only pass through the maximum value, so only a small part of data in the T-F domain are used to update the parameters in the neural network. Because of the maximum selection strategy, GMP encourages only one point in a T-F segmentation mask to be positive, so GMP will underestimate [23] the sound events in the T-F representation. Examples of T-F segmentation masks learned using GMP are shown in Fig. 7(c).

2) Global average pooling: Global average pooling (GAP) was first applied in image classification [31]. GAP on each T-F segmentation mask $h_{k}$ is depicted as:

$$
F\left(h_{k}\right)=\frac{1}{T F} \sum_{t}^{T} \sum_{f}^{F} h_{k}(t, f) .
$$

GAP corresponds to the collective assumption in MIL [48], which states that all T-F units in a T-F segmentation mask contribute equally to the label of an audio clip. That is, all T$\mathrm{F}$ units in a T-F segmentation mask are assumed to contain the labelled sound events. However, some sound events only last a short time, so GAP usually overestimates the sound events [31]. Examples of T-F segmentation masks learned using GAP are shown in Fig 7(d).

3) Global weighted rank pooling: To overcome the limitations of GMP and GAP, which underestimate and overestimate the sound events in the T-F segmentation masks, global weighted rank pooling (GWRP) is proposed in [23]. GWRP can be seen as a generalization of GMP and GAP. The idea of GWRP is to put a descending weight on the values of a T-F segmentation mask sorted in a descending order. Let an index set $I^{c}=\left\{i_{1}, \ldots i_{M}\right\}$ define the descending order of the values within a T-F segmentation mask $h_{k}$, i.e. $\left(h_{k}\right)_{i_{1}} \geq\left(h_{k}\right)_{i_{2}} \geq \ldots \geq\left(h_{k}\right)_{i_{n}}$, where $M=T \times F$ is the number of T-F units in a T-F segmentation mask. Then the GWRP is defined as:

$$
F\left(h_{k}\right)=\frac{1}{Z(r)} \sum_{j=1}^{M} r^{j-1}\left(h_{k}\right)_{i_{j}},
$$



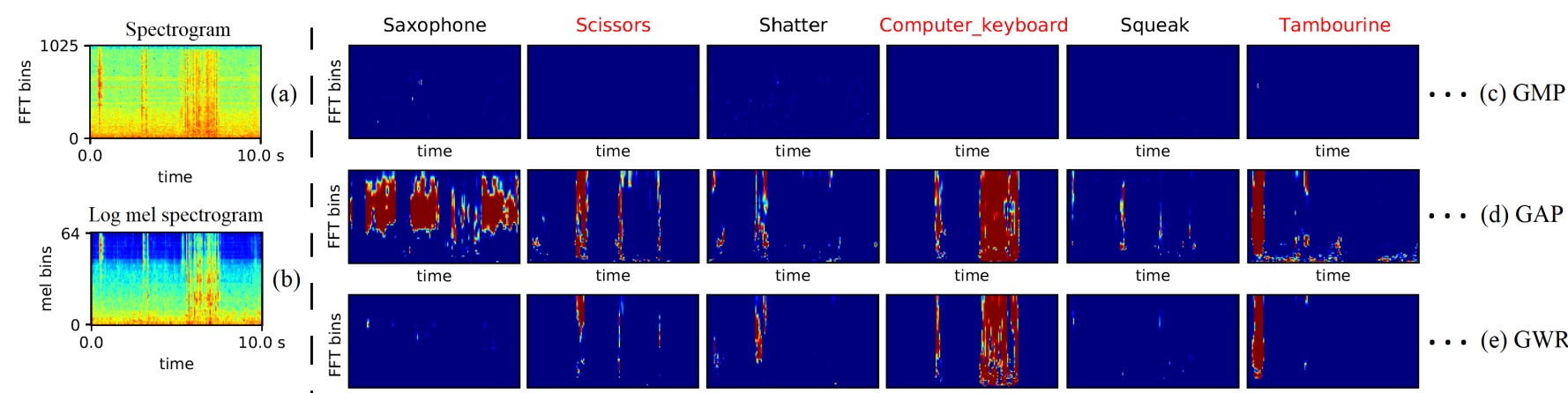

time
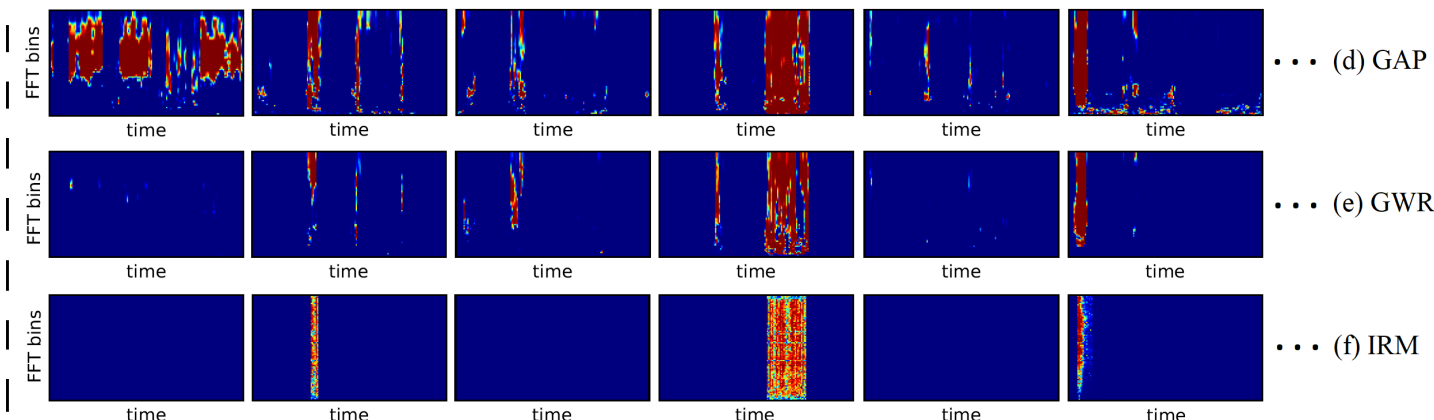

time

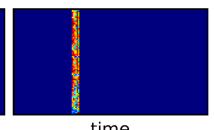

time

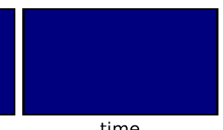

time

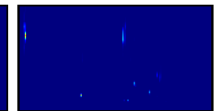

time

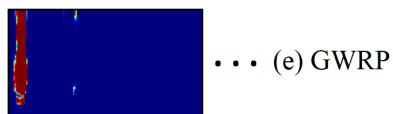

time
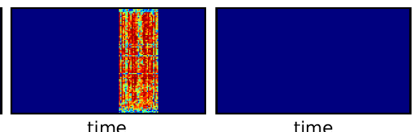

time

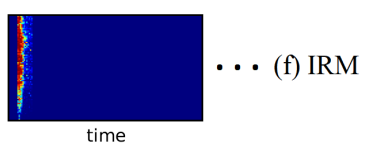

Fig. 7. (a) Spectrogram of an audio clip containing "scissors", "computer keyboard" and "tambourine" (plotted in log scale); (b) Log mel spectrogram of the audio clip; (c) Upsampled T-F segmentation masks $\widetilde{h}_{k}$ of sound events learned using global max pooling (GMP). Only a few T-F units have high value and the other parts of the T-F segmentation masks are dark; (d) Upsampled T-F segmentation masks $\widetilde{h}_{k}$ of sound events learned using global average pooling (GAP); (e) Upsampled T-F segmentation masks $\widetilde{h}_{k}$ of sound events learned using global weighted rank pooling (GWRP); (f) Ideal ratio mask (IRM) of sound events. Only 6 out of 41 T-F segmentation masks are plotted due to the limited space.

where $0 \leq r \leq 1$ is a hyper parameter and $Z(r)=\sum_{j=1}^{M} r^{j-1}$ is a normalization term. When $r=0$ GWRP becomes GMP and when $r=1$ GWRP becomes GAP. The hyperparameter $r$ can vary depending on the frequency of occurrence of the sound events. GWAP attends more to the T-F units of high values in a T-F segmentation mask and less to those of low values in a T-F segmentation mask. The T-F segmentation masks learned using GWMP is shown in Fig. 7(e). The ideal binary masks (IBMs) of the sound events are plotted in Fig. 7(f) for comparison with the GMP, GAP and GWRP.

\section{Post-processing for sound event detection}

In Section III-C we mentioned that the frame-wise scores $v_{k}(t)$ can be obtained from the T-F segmentation masks using Equation (2). To reduce the number of false alarms, for an audio clip, we only apply sound event detection on the sound classes with positive audio tagging predictions. Then we apply thresholds on the frame-wise predictions $v_{k}(t)$ to obtain the event-wise predictions. We apply a high threshold of 0.2 to detect the presence of sound events and then extend the boundary of both onset and offset sides until the frame-wise scores drop below threshold of 0.1 . This two-step threshold method will produce smooth predictions of sound events. As the duration of sound events in DCASE 2018 Task 2 varies from $300 \mathrm{~ms}$ to $30 \mathrm{~s}$, we remove the detected sound events that are shorter than $320 \mathrm{~ms}$ (10 frames) to reduce false alarms and join the sound events whose silence gap is shorter than $320 \mathrm{~ms}$ (10 frames).

\section{EXPERIMENTS}

\section{A. Dataset}

We mix the DCASE 2018 Task 1 acoustic scene dataset [49] with the DCASE 2018 Task 2 general-purpose Freesound dataset [50] under different signal-to-noise ratios (SNRs) to evaluate the proposed methods. The reason for this choice is that DCASE 2018 Task 1 provides background sounds recorded from a variety of real world scenes whereas the DCASE 2018 Task 2 provides a variety of foreground sound events. The DCASE 2018 Task 1 contains 8640 10-second audio clips in the development set of subtask A. The audio clips are recorded from 10 different scenes such as "airport", "metro station" and "urban park". The DCASE 2018 Task 2 contains 3710 manually verified sound events ranging in length from $300 \mathrm{~ms}$ to $30 \mathrm{~s}$ depending on the audio classes. There are 41 classes of sound events such as "flute", "applause" and "cough". We only use these manually verified audio clips from the DCASE 2018 Task 2 as sound events because the remaining audio clips are unverified and may contain noisy labels. We truncated the sound events to up to 2 seconds and mix them with the 10-second audio clips from the DCASE 2018 Task 1 acoustic scene dataset. The mixed audio clips are single channel with a sampling rate of $32 \mathrm{kHz}$. Each mixed audio clip contains three non-overlapped sound events. We mixed the sound events with the acoustic scenes for SNRs at $20 \mathrm{~dB}, 10 \mathrm{~dB}$ and $0 \mathrm{~dB}$. For each SNR, the 8000 mixed audio clips are divided into 4 cross-validation folds. Fig. 7(b) shows the log mel spectrogram of a mixed 10-second audio clip. The source code of our work is released ${ }^{1}$.

\section{B. Evaluation metrics}

We use F-score [51], area under the curve (AUC) [52] and mean average precision (mAP) [6] in the evaluation of the audio tagging, the frame-wise SED and the T-F segmentation. We also use error rate (ER) for evaluating the event-wise SED.

1) Basic statistics: True positive (TP): Both the reference and the system prediction indicate an event to be active. False negative (FN): The reference indicates an event to be active but the system prediction indicates an event to be inactive. False positive (FP): The system prediction indicates an event to be active but the reference indicates it is not [51].

\footnotetext{
${ }^{1}$ https://github.com/qiuqiangkong/sed_time_freq_segmentation
} 
2) Precision, recall and F-score: Precision $(P)$ and recall $(R)$ are defined as [51]:

$$
P=\frac{T P}{T P+F P}, \quad R=\frac{T P}{T P+F N} .
$$

Bigger $P$ and $R$ indicates better performance. F-score is calculated based on $P$ and $R$ [51]:

$$
F=\frac{2 P \cdot R}{P+R}=\frac{T P}{T P+(F N+F P) / 2} .
$$

Bigger F-score indicates better performance.

3) Area under the curve (AUC): A receiver operating characteristic (ROC) curve [52] plots true positive rate (TPR) versus false positive rate (FPR). Area under the curve (AUC) score is the area under this ROC curve which summarizes the ROC curve to a single number. Using the AUC does not require manual selection of a threshold. Bigger AUC indicates better performance. A random guess has an AUC of 0.5.

4) Average precision: Average precision (AP) is the average of the precision at different recall values. Similar to AUC, AP does not rely on the threshold. Different to AUC, AP does not count the true negatives and is widely used as a criterion in imbalanced dataset such as object detection [6].

5) Error rate: Error rate (ER) is an event-wise evaluation metric. ER measures the amount of errors in terms of insertions (I), deletions (D) and substitutions (S) [51]. For an audio clip, the insertions, deletions and substitutions are defined as:

$$
\begin{aligned}
S & =\min (F N, F P), \\
D & =\max (0, F N-F P), \\
I & =\max (0, F P-F N),
\end{aligned}
$$

where FN, FP, FN are event-wise statistics in an audio clip. Lower $E R, S, D$ and $I$ indicate the better performance. When evaluating the event based criterion, we allow some degree of misalignment between a reference and a system output for counting a true positive $[12,51,53]$. Following the default configuration of [51], we adopt an onset collar of $200 \mathrm{~ms}$ and an offset collar of $200 \mathrm{~ms} / 50 \%$ to count the true positive of a detection. We used the toolbox [51] for evaluating the performance of the event-based SED.

\section{Feature extraction}

We apply a fast Fourier transform (FFT) with a window size of 2048 and an overlap of 1024 between neighbouring windows to extract the spectrogram of audio clips. This configuration that follows [54] offers a good resolution in both time and frequency domain. Then mel filter banks with 64 bands are applied on the spectrogram followed by logarithm operation to obtain $\log$ mel spectrogram as the input T-F representation feature. Log mel spectrogram has been widely used in audio classification [28, 54].

\section{Model}

In this subsection we give a detailed description of the configuration of the segmentation mapping in Section IV-A and the classification mapping in Section IV-B. We apply a
TABLE I

\begin{tabular}{|c|c|c|c|c|c|c|c|c|c|}
\hline \multirow[b]{2}{*}{ Algorithms } & \multicolumn{3}{|c|}{$20 \mathrm{~dB}$} & \multicolumn{3}{|c|}{$10 \mathrm{~dB}$} & \multicolumn{3}{|c|}{$\mathbf{O} \mathrm{dB}$} \\
\hline & F1 & AUC & $\mathrm{mAP}$ & F1 & AUC & $\mathrm{mAP}$ & F1 & AUC & $\mathrm{mAP}$ \\
\hline DNN [55] & 0.439 & 0.885 & 0.468 & 0.396 & 0.861 & 0.402 & 0.331 & 0.810 & 0.314 \\
\hline WLD CNN [37] & 0.498 & 0.777 & 0.498 & 0.524 & 0.794 & 0.526 & 0.528 & 0.815 & 0.535 \\
\hline FrameCNN [34] & 0.581 & 0.899 & 0.587 & 0.543 & 0.883 & 0.526 & 0.484 & 0.850 & 0.439 \\
\hline Attention [39] & 0.714 & 0.922 & 0.755 & 0.690 & 0.907 & 0.729 & 0.612 & 0.875 & 0.643 \\
\hline GMP & 0.435 & 0.818 & 0.475 & 0.406 & 0.801 & 0.440 & 0.373 & 0.773 & 0.389 \\
\hline GAP & 0.529 & 0.934 & 0.623 & 0.467 & 0.914 & 0.555 & 0.385 & 0.877 & 0.442 \\
\hline GWRP & 0.635 & 0.955 & 0.753 & 0.604 & 0.942 & 0.696 & 0.534 & 0.915 & 0.596 \\
\hline
\end{tabular}

CONFIGURATION OF CNN.

\begin{tabular}{c|c}
\hline Layers & $\begin{array}{c}\text { Output size } \\
\text { (feature maps } \times \text { time steps } \times \text { mel bins) }\end{array}$ \\
\hline Input log mel spectrogram & $1 \times 311 \times 64$ \\
\hline$\{3 \times 3,32, \mathrm{BN}$, ReLU $\} \times 2$ & $32 \times 311 \times 64$ \\
\hline$\{3 \times 3,64, \mathrm{BN}, \mathrm{ReLU}\} \times 2$ & $64 \times 311 \times 64$ \\
\hline$\{3 \times 3,128, \mathrm{BN}, \mathrm{ReLU}\} \times 2$ & $128 \times 311 \times 64$ \\
\hline$\{3 \times 3,128, \mathrm{BN}, \mathrm{ReLU}\} \times 2$ & $128 \times 311 \times 64$ \\
\hline $1 \times 1,41$, sigmoid & $41 \times 311 \times 64$ \\
\hline Global pooling $(\mathrm{GP})$ & 41 \\
\hline
\end{tabular}

TABLE II

F1-SCORE, AUC AND MAP OF AUDIO TAGGING AT DIFFERENT SNRS.

"VGG-like" convolutional neural network [56] with 8 convolutional blocks on the input log mel spectrogram [54]. Each convolutional layer consists of a linear convolution with a filter size of $3 \times 3$ followed by a batch normalization layer [43] and a ReLU activation function [44]. We use 4 convolution blocks following the baseline system of DCASE 2018 [54]. The number of feature maps of the convolutional layers are 32,64, 128 and 128, respectively. This configuration is to fit the model to a single GPU card with 12 GB RAM sufficiently. Then a $1 \times 1$ convolutional layer with sigmoid non-linearity is applied to convert the feature maps to the T-F segmentation masks of sound events. Then a global pooling is used to summarize each T-F segmentation mask to a scalar representing the presence probability of the sound events in an audio clip. We summarize the configuration of the neural network in Table I. In training we use a mini-batch size of 24 to fully utilize the single card GPU with 12 GB RAM. The Adam optimizer [57] with a learning rate 0.001 is used for its fast convergence.

\section{E. Audio tagging}

We compare our method with fully connected neural network [55], CNN trained on weakly labelled data [37], FrameCNN [34] and the attention model [39]. We apply GMP, GAP and GWRP as global pooling in our model. Table II shows that for SNR at $20 \mathrm{~dB}$, the attention model [39] achieves the best F1-score of 0.714 and mAP of 0.755 followed by the GWRP of 0.635 and 0.753 , respectively. On the other hand, GWRP achieves the best AUC of 0.955. Comparing the performance under different SNRs, the F1-score and mAP drop approximately 0.1 in absolute value for SNR changed from $20 \mathrm{~dB}$ to $0 \mathrm{~dB}$. AUC drop approximately 0.04 in absolute 
TABLE III

F1-SCORE OF AUDIO TAGGING AT 0 DB SNR

\begin{tabular}{|c|c|c|c|c|c|c|c|c|c|c|c|c|c|c|c|c|c|c|c|c|c|}
\hline & $\begin{array}{l}\text { Acous. } \\
\text { guitar }\end{array}$ & $\begin{array}{l}\text { Appla- } \\
\text { use }\end{array}$ & Bark & $\begin{array}{l}\text { Bass } \\
\text { drum }\end{array}$ & $\begin{array}{l}\text { Burp- } \\
\text { ing }\end{array}$ & Bus & Cello & Chime & $\begin{array}{l}\text { Clari- } \\
\text { net }\end{array}$ & $\begin{array}{l}\text { Keybo- } \\
\text { ard }\end{array}$ & Cough & $\begin{array}{l}\text { Cow- } \\
\text { bell }\end{array}$ & $\begin{array}{l}\text { Double } \\
\text { bass }\end{array}$ & Drawer & $\begin{array}{l}\text { Elec. } \\
\text { piano }\end{array}$ & Fart & $\begin{array}{l}\text { Finger } \\
\text { snap }\end{array}$ & $\begin{array}{l}\text { Fire- } \\
\text { works }\end{array}$ & Flute & $\begin{array}{l}\text { Glock- } \\
\text { enspiel }\end{array}$ & Gong \\
\hline DNN [55] & 0.286 & 0.873 & 0.332 & 0.041 & 0.344 & 0.367 & 0.489 & 0.546 & 0.423 & 0.283 & 0.075 & 0.133 & 0.197 & 0.083 & 0.304 & 0.267 & 0.389 & 0.285 & 0.350 & 0.464 & 0.310 \\
\hline NN [37] & 633 & 0.896 & 0.719 & 0.547 & 0.794 & 0.248 & 0.610 & 0.589 & 0.504 & 0.390 & 0.513 & 0.889 & 0.436 & 0.136 & 0.435 & 0.384 & 0.672 & 0.375 & 0.270 & 0.692 & 0.513 \\
\hline FrameCNN [34] & 0.416 & 0.878 & 0.719 & 0.166 & 0.557 & 0.385 & 0.529 & 0.562 & 0.448 & 0.507 & 0.484 & 0.668 & 0.314 & 0.181 & 0.392 & 0.304 & 0.556 & 474 & 0.385 & 0.488 & 0.465 \\
\hline Attention [39] & 0.548 & 0.893 & 0.761 & 0.632 & 0.866 & 0.335 & 0.616 & 0.607 & 0.568 & 0.497 & 0.565 & 0.924 & 0.477 & 0.160 & 0.546 & 0.598 & 0.823 & 0.463 & 0.565 & 0.901 & 0.617 \\
\hline GMP & 0.458 & 0.522 & 0.335 & 0.183 & 0.400 & 0.087 & 0.299 & 0.468 & 0.424 & 0.422 & 0.151 & 0.774 & 0.281 & 0.076 & 0.279 & 0.284 & 0.176 & 0.271 & 0.315 & 0.844 & 0.434 \\
\hline GAP & 0.547 & 0.817 & 0.409 & .070 & 0.484 & 0.205 & 0.435 & 0.501 & 0.354 & 0.504 & 0.347 & 0.314 & 0.181 & 0.164 & 0.218 & 0.407 & 0.399 & 0.346 & 0.343 & 0.496 & 0.305 \\
\hline \multirow[t]{2}{*}{ GWRP } & 0.552 & 0.825 & 0.654 & 0.204 & 0.578 & 0.342 & 0.416 & 0.628 & 0.424 & 0.573 & 0.543 & 0.579 & 0.333 & 0.320 & 0.421 & 0.618 & 0.473 & 0.558 & 0.427 & 0.726 & 0.550 \\
\hline & hot & $\begin{array}{l}\text { Harmo- } \\
\text { nica }\end{array}$ & $\begin{array}{l}\mathrm{Hi}- \\
\text { hat }\end{array}$ & Key & Knock & $\begin{array}{l}\text { Laugh- } \\
\text { ter }\end{array}$ & Meow & $\begin{array}{l}\text { Micro- } \\
\text { wave }\end{array}$ & Oboe & $\begin{array}{l}\text { Saxo- } \\
\text { phone }\end{array}$ & $\begin{array}{l}\text { Sciss- } \\
\text { ors }\end{array}$ & Shatter & $\begin{array}{l}\text { Snare } \\
\text { drum }\end{array}$ & Squeak & $\begin{array}{l}\text { Tambo- } \\
\text { urine }\end{array}$ & $\begin{array}{l}\text { Tear- } \\
\text { ing }\end{array}$ & $\begin{array}{l}\text { Tele- } \\
\text { phone }\end{array}$ & \multicolumn{2}{|c|}{ Trumpet Violin } & $\begin{array}{l}\text { Writ- } \\
\text { ing }\end{array}$ & vg. \\
\hline DNN [55] & 0.297 & 0.672 & 0.547 & 0.418 & 0.276 & 0.192 & 0.075 & 0.121 & 0.408 & 0.500 & 0.411 & 0.336 & 0.368 & 0.097 & 0.299 & 0.254 & 0.270 & 0.528 & 0.379 & 0.293 & 0.331 \\
\hline WLD CNN & 0.538 & 0.742 & 0.910 & 0.643 & 0.649 & 0.361 & 0.359 & 0.263 & 0.5 & 0.636 & 0.558 & 0.410 & 0.599 & 0.052 & 0.593 & 0.436 & 0.324 & & 0.755 & & 0.528 \\
\hline FrameCNN [34] & 0.424 & 0.723 & 0.688 & 0.660 & 0.553 & 0.390 & 0.355 & 0.400 & 0.490 & 0.528 & 0.497 & 0.481 & 0.624 & 0.193 & 0.733 & 0.449 & 0.346 & 0.526 & 0.475 & 0.431 & 0.484 \\
\hline Attention [39] & 0.607 & 0.759 & 0.938 & 0.744 & 0.738 & 0.444 & 0.499 & 0.441 & 0.560 & 0.678 & 0.660 & 0.693 & 0.709 & 0.113 & 0.957 & 0.593 & 0.434 & 0.368 & 0.784 & 0.400 & 0.612 \\
\hline GMP & 0.398 & 0.322 & 0.796 & 0.141 & 0.483 & 0.311 & 0.275 & 0.207 & 0.442 & 0.474 & 0.173 & 0.251 & 0.465 & 0.031 & 0.891 & 0.504 & 0.329 & 0.585 & 0.567 & 0.175 & 0.373 \\
\hline O & & 0.681 & 0.641 & 0.392 & 0.402 & 0.480 & 0.203 & 0.172 & & 0. & 0.404 & 0.392 & 0.335 & 0.161 & 0.412 & 0.348 & 0.341 & 0.579 & 0.349 & 0.408 & 0.385 \\
\hline GWRP & 0.523 & 0.714 & 0.798 & 0.606 & 0.524 & 0.563 & 0.547 & 0.353 & 0.487 & 0.534 & 0.452 & 0.653 & 0.585 & 0.260 & 0.857 & 0.583 & 0.508 & 0.639 & 0.516 & 0.452 & 0.534 \\
\hline
\end{tabular}

TABLE IV

F1-SCORE OF FRAME-WISE SED AT 0 DB SNR.

\begin{tabular}{|c|c|c|c|c|c|c|c|c|c|c|c|c|c|c|c|c|c|c|c|c|c|}
\hline & $\begin{array}{l}\text { Acous. } \\
\text { guitar }\end{array}$ & $\begin{array}{l}\text { Appla- } \\
\text { use }\end{array}$ & Bark & $\begin{array}{l}\text { Bass } \\
\text { drum }\end{array}$ & $\begin{array}{l}\text { Burp- } \\
\text { ing }\end{array}$ & us & o & ime & $\begin{array}{l}\text { Clari- } \\
\text { net }\end{array}$ & $\begin{array}{l}\text { Keybo- } \\
\text { ard }\end{array}$ & Cough & $\begin{array}{l}\text { Cow- } \\
\text { bell }\end{array}$ & $\begin{array}{l}\text { Double } \\
\text { bass }\end{array}$ & Drawer & $\begin{array}{l}\text { Elec. } \\
\text { piano }\end{array}$ & Fart & $\begin{array}{l}\text { Finger } \\
\text { snap }\end{array}$ & $\begin{array}{l}\text { Fire- } \\
\text { works }\end{array}$ & lute & $\begin{array}{l}\text { Glock- } \\
\text { enspiel }\end{array}$ & ong \\
\hline DNN & 1 & 0.746 & 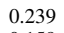 & 9 & 0.317 & 0.306 & & 0.495 & 5 & 0.202 & 0 & 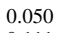 & 0 & 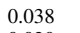 & 3 & 97 & 6 & 95 & 214 & .291 & .212 \\
\hline$n$ & & & & & & & & & & & & & & & & & & & & & .037 \\
\hline Fram & 294 & 741 & 0.585 & 07 & 411 & & 0.441 & .480 & 0.342 & & 0.370 & 283 & 0.178 & 0.102 & 0.310 & 39 & 6 & 25 & 16 & & 308 \\
\hline Atten & 062 & 0.422 & 0.069 & 0.020 & 189 & 0.024 & 0.242 & 0.263 & 0.210 & 019 & 0.059 & .051 & 0.045 & 0.003 & 0.068 & 0.050 & 0.076 & 0.031 & .159 & 0.026 & 0.088 \\
\hline GN & & & 0.000 & & & & & & & & & & & & & & & & & & 0.000 \\
\hline & & & & & & & & & & & & & & & & & & & & & 0.200 \\
\hline \multirow[t]{2}{*}{ GWRP } & 153 & 0.704 & 0.507 & 0.072 & 0.456 & 0.188 & 0.326 & 0.575 & 0.341 & 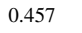 & 0.402 & 222 & 0.193 & 0.172 & 0.351 & 0.498 & 0.247 & 0.355 & 0.316 & .596 & 0.418 \\
\hline & & $\begin{array}{l}\text { Harmo- } \\
\text { nica }\end{array}$ & $\begin{array}{l}\text { Hi- } \\
\text { hat }\end{array}$ & Keys & $\mathrm{k}$ & $\begin{array}{l}\text { Laugh- } \\
\text { ter }\end{array}$ & $\mathrm{N}$ & $\begin{array}{l}\text { Micro- } \\
\text { wave }\end{array}$ & o & phone & $\begin{array}{l}\text { Sciss- } \\
\text { ors }\end{array}$ & er & $\begin{array}{l}\text { Snare } \\
\text { drum }\end{array}$ & ak & $\begin{array}{l}\text { Tambo- } \\
\text { urine }\end{array}$ & $\begin{array}{l}\text { Tear- } \\
\text { ing }\end{array}$ & $\begin{array}{l}\text { Tele- } \\
\text { phone }\end{array}$ & \multicolumn{2}{|c|}{ Trumpet Violin } & $\begin{array}{l}\text { Writ- } \\
\text { ing }\end{array}$ & vg. \\
\hline & & 0.594 & 0.510 & & & 0.111 & & 0.095 & & 0317 & 0.277 & & 0 & & & & & & & & 0.237 \\
\hline & & & & & & & & & & & & & & & & & & & & & 0.140 \\
\hline Fram & 0.259 & (1.07 & 0.639 & 0.495 & & 0.271 & 0.228 & 0.284 & & & 0.379 & & 0.453 & 0.111 & & 0.277 & & 0.407 & 0.228 & .299 & 0.343 \\
\hline Attention [39] & 0.029 & 0.143 & 0.107 & 0.096 & 0.101 & 0.051 & 0.034 & 0.018 & 0.137 & 0.353 & 0.078 & 0.038 & 0.054 & 0.005 & 0.188 & 0.046 & 0.148 & 0.08 & 0.156 & 0.056 & 0.100 \\
\hline & & & & & & & & & & & & & & & & & & & & & .000 \\
\hline & & & & & & & & & & & & & & & & & & & & & 0.252 \\
\hline GWRP & 0.362 & 0.649 & 0.696 & 0.539 & 0.354 & 0.429 & 0.400 & 0.182 & 0.404 & 0.440 & 0.384 & 0.471 & 0.373 & 0.173 & 0.591 & 0.378 & 0.420 & 0.528 & 0.331 & 0.360 & 0.398 \\
\hline
\end{tabular}

TABLE V

F1-SCORE, AUC AND MAP OF FRAME-WISE SED AT DIFFERENT SNRS.

\begin{tabular}{|c|c|c|c|c|c|c|c|c|c|}
\hline \multirow[b]{2}{*}{ Algorithms } & \multicolumn{3}{|c|}{$20 \mathrm{~dB}$} & \multicolumn{3}{|c|}{$10 \mathrm{~dB}$} & \multicolumn{3}{|c|}{$\mathbf{0 ~ d B}$} \\
\hline & $\mathrm{F} 1$ & AUC & $\mathrm{mAP}$ & $\mathrm{F} 1$ & AUC & $\mathrm{mAP}$ & F1 & AUC & $\mathrm{mAP}$ \\
\hline DNN [55] & 0.360 & 0.722 & 0.269 & 0.306 & 0.702 & 0.224 & 0.237 & 0.666 & 0.169 \\
\hline WLD CNN [37] & 0.168 & 0.669 & 0.179 & 0.182 & 0.688 & 0.201 & 0.140 & 0.701 & 0.166 \\
\hline FrameCNN [34] & 0.440 & 0.808 & 0.369 & 0.399 & 0.787 & 0.329 & 0.343 & 0.756 & 0.275 \\
\hline Attention [39] & 0.163 & 0.827 & 0.317 & 0.137 & 0.807 & 0.278 & 0.100 & 0.773 & 0.221 \\
\hline GMP & 0.000 & 0.676 & 0.090 & 0.000 & 0.658 & 0.076 & 0.000 & 0.649 & 0.072 \\
\hline GAP & 0.398 & 0.790 & 0.400 & 0.334 & 0.753 & 0.328 & 0.252 & 0.712 & 0.245 \\
\hline GWRP & 0.511 & 0.886 & 0.508 & 0.472 & 0.871 & 0.453 & 0.398 & 0.829 & 0.360 \\
\hline
\end{tabular}

TABLE VI

F1-SCORE, AUC AND MAP OF EVENT-WISE SED AT DIFFERENT SNRS.

\begin{tabular}{|c|c|c|c|c|c|c|c|c|c|c|c|c|}
\hline \multirow[b]{2}{*}{ Algorithms } & \multicolumn{4}{|c|}{$20 \mathrm{~dB}$} & \multicolumn{4}{|c|}{$10 \mathrm{~dB}$} & \multicolumn{4}{|c|}{$0 \mathrm{~dB}$} \\
\hline & F1 & ER & D & I & F1 & ER & D & I & F1 & ER & D & I \\
\hline DNN [55] & 0.226 & 1.91 & 0.75 & 1.16 & 0.178 & 2.29 & 0.79 & 1.50 & 0.120 & 2.80 & 0.84 & 1.96 \\
\hline WLD CNN [37] & 0.010 & 1.16 & 0.99 & 0.17 & 0.011 & 1.15 & 0.99 & 0.17 & 0.018 & 1.12 & 0.99 & 0.13 \\
\hline FrameCNN [34] & 0.166 & 2.38 & 0.79 & 1.58 & 0.151 & 2.49 & 0.81 & 1.68 & 0.141 & 2.70 & 0.81 & 1.88 \\
\hline Attention [39] & 0.028 & 1.10 & 0.96 & 0.14 & 0.021 & 1.10 & 0.97 & 0.13 & 0.011 & 1.09 & 0.98 & 0.10 \\
\hline GMP & 0.000 & 1.00 & 1.00 & 0.00 & 0.000 & 1.00 & 1.00 & 0.00 & 0.000 & 1.00 & 1.00 & 0.00 \\
\hline GAP & 0.173 & 2.71 & 0.78 & 1.93 & 0.139 & 2.95 & 0.82 & 2.13 & 0.098 & 3.52 & 0.86 & 2.66 \\
\hline GWRP & 0.254 & 2.12 & 0.66 & 1.45 & 0.227 & 2.30 & 0.69 & 1.61 & 0.167 & 2.55 & 0.76 & 1.78 \\
\hline
\end{tabular}

value for SNR changed from $20 \mathrm{~dB}$ to $0 \mathrm{~dB}$. This result shows that there is a large variance in audio tagging under low SNR. Table III shows the audio tagging results of all sound events under $0 \mathrm{~dB}$ SNR. Some sound events such as "hi-hat" and "tambourine" have higher classification accuracy while some sound events such as "microwave" and "squeak" are difficult to recognize. On average, the attention model [39] achieves the best F1-score of 0.612 followed by GWRP of 0.534 .

\section{F. Frame-wise sound event detection}

Table IV shows the F1-score of the frame-wise SED for all sound classes under SNR of $0 \mathrm{~dB}$. GWRP achieves the best averaged F1-score of 0.398 , followed by the FrameCNN model [34] of 0.343. Some classes such as "applause" and "hi-hat" have higher F1-score by the frame-wise SED, while some classes such as "drawer" and squeak" have lower F1score by the frame-wise SED. Table V shows the frame-wise SED results under different SNRs. GWRP achieves the best F1-score, AUC and mAP of 0.511, 0.886 and 0.508 under $20 \mathrm{~dB}$ SNR. The FrameCNN model [34] achieves a second place with an F1-score of 0.440. GAP overestimates the sound events which is shown in the visualization of the upsampled TF segmentation masks (Fig. 7). GAP does not perform better than GWRP. GMP underestimates the sound events (Fig. 7) and performs worst in frame-wise SED. In GWRP, the F1score drops from 0.511 to 0.472 to 0.398 under SNRs of 20 $\mathrm{dB}, 10 \mathrm{~dB}$ and $0 \mathrm{~dB}$. Fig. 8 shows the frame-wise scores of sound events obtained from equation (2) under SNR of $0 \mathrm{~dB}$. Frame-wise scores obtained by using GWRP looks 
TABLE VII

F1-SCORE OF EVENT-WISE SED AT 0 DB SNR.

\begin{tabular}{|c|c|c|c|c|c|c|c|c|c|c|c|c|c|c|c|c|c|c|c|c|c|}
\hline & $\begin{array}{l}\text { Acous. } \\
\text { guitar }\end{array}$ & $\begin{array}{l}\text { Appla- } \\
\text { use }\end{array}$ & Bark & $\begin{array}{l}\text { Bass } \\
\text { drum }\end{array}$ & $\begin{array}{l}\text { Burp- } \\
\text { ing }\end{array}$ & Bus & Cello & hime & $\begin{array}{l}\text { Clari- } \\
\text { net }\end{array}$ & $\begin{array}{l}\text { Keybo- } \\
\text { ard }\end{array}$ & Cough & $\begin{array}{l}\text { Cow- } \\
\text { bell }\end{array}$ & $\begin{array}{l}\text { Double } \\
\text { bass }\end{array}$ & Drawer & $\begin{array}{l}\text { Elec. } \\
\text { piano }\end{array}$ & Fart & $\begin{array}{l}\text { Finger } \\
\text { snap }\end{array}$ & $\begin{array}{l}\text { Fire- } \\
\text { works }\end{array}$ & Flute & $\begin{array}{l}\text { Glock- } \\
\text { enspiel }\end{array}$ & Gong \\
\hline DNN & & 87 & 0 & 2 & & & & & & & & 07 & 0 & 050 & 0 . & & 5 & 13 & 36 & 79 & 0.159 \\
\hline$w$ & & & & & & & & & & & & & & 001 & & & & & & 1 & .001 \\
\hline Fran & 98 & 10 & 187 & 12 & 90 & 30 & 287 & & 57 & 4 & 44 & 005 & 0.04 & 0.091 & 68 & & & 33 & 81 & 265 & .098 \\
\hline Attent & 000 & 0.051 & 0.000 & 0.000 & 0.052 & 0.000 & 0.020 & 0.018 & 0.000 & .000 & 0.000 & 0.000 & 0.009 & 0.000 & 0.000 & 0.000 & 0.000 & 0.000 & 0.019 & 0.000 & 0.000 \\
\hline GMP & 000 & 00 & 0.000 & 00 & 0 & 0 & 00 & 0.000 & 00 & 0.000 & 0. & 000 & 0.0 & 0 & 0. & 0 & 0 & 0 & 00 & 0.000 & 0.000 \\
\hline & & & 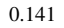 & & & & & & & & & & & & & & & & & & 0.032 \\
\hline \multirow[t]{2}{*}{ GWRP } & 31 & 225 & 0.315 & 002 & 352 & .030 & .086 & 363 & 0.086 & 211 & 0.228 & .010 & 0.089 & 0.111 & 0.153 & 0.312 & .068 & 0.144 & .060 & .004 & 0.281 \\
\hline & Gunshot & $\begin{array}{l}\text { Harmo } \\
\text { nica }\end{array}$ & $\begin{array}{l}\text { Hi- } \\
\text { hat }\end{array}$ & Keys & lock & $\begin{array}{l}\text { Laugh- } \\
\text { ter }\end{array}$ & $\mathrm{N}$ & $\begin{array}{l}\text { Micro- } \\
\text { wave }\end{array}$ & Oboe & $\begin{array}{l}\text { Saxo- } \\
\text { phone }\end{array}$ & $\begin{array}{l}\text { Sciss- } \\
\text { ors }\end{array}$ & er & $\begin{array}{l}\text { Snare } \\
\text { drum }\end{array}$ & ak & $\begin{array}{l}\text { Tambo- } \\
\text { urine }\end{array}$ & $\begin{array}{l}\text { Tear- } \\
\text { ing }\end{array}$ & $\begin{array}{l}\text { Tele- } \\
\text { phone }\end{array}$ & \multicolumn{2}{|c|}{ Trumpet Violin } & $\begin{array}{l}\text { Writ- } \\
\text { ing }\end{array}$ & vg. \\
\hline D & & 455 & 0.20 & & & 0054 & & 0047 & & & 0. & & 0. & & & & 0.100 & & 1 & & 0.120 \\
\hline & & & & & & & & & & & & & & & & & & & & & 0.018 \\
\hline & & 20 & 0.409 & & & 3 & & & & & 0.140 & & & & & & 0.071 & & 0.052 & & 0.141 \\
\hline Attention [39] & 0.000 & 0.000 & 0.000 & 0.000 & 0.106 & 0.000 & 0.000 & 0.000 & 0.000 & 0.188 & 0.000 & 0.000 & 0.000 & 0.000 & 0.000 & 0.000 & 0.000 & 0.000 & 0.000 & 0.000 & 0.011 \\
\hline GM & & & 0.000 & & & 000 & 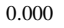 & & & & 0. & & 0 & & & 0. & & & 00 & 0.000 & 0.000 \\
\hline & & & & & & & & & & & & & & & & & & & & & \\
\hline GWRP & 0.078 & 0.519 & 0.031 & 356 & 0.206 & 0.205 & 0.269 & 0.118 & 0.252 & 165 & 0.167 & 0.130 & 0.065 & 0.067 & 0.065 & 0.146 & 0.238 & 0.175 & 0.105 & 0.243 & 0.167 \\
\hline
\end{tabular}

TABLE VIII

F1-SCORE OF TIME-FREQUENCY SEGMENTATION AT 0 DB SNR.

\begin{tabular}{|c|c|c|c|c|c|c|c|c|c|c|c|c|c|c|c|c|c|c|c|c|c|}
\hline & $\begin{array}{l}\text { Acous. } \\
\text { guitar }\end{array}$ & $\begin{array}{l}\text { Appla- } \\
\text { use }\end{array}$ & Bark & $\begin{array}{l}\text { Bass } \\
\text { drum }\end{array}$ & $\begin{array}{l}\text { Burp- } \\
\text { ing }\end{array}$ & Bus & Cello & Chime & $\begin{array}{l}\text { Clari- } \\
\text { net }\end{array}$ & $\begin{array}{l}\text { Keybo- } \\
\text { ard }\end{array}$ & Cough & $\begin{array}{l}\text { Cow- } \\
\text { bell }\end{array}$ & $\begin{array}{l}\text { Double } \\
\text { bass }\end{array}$ & Drawer & $\begin{array}{l}\text { Elec. } \\
\text { piano }\end{array}$ & Fart & $\begin{array}{l}\text { Finger } \\
\text { snap }\end{array}$ & $\begin{array}{l}\text { Fire- } \\
\text { works }\end{array}$ & Flute & $\begin{array}{l}\text { Glock- } \\
\text { enspiel }\end{array}$ & Gong \\
\hline GMP & 0.000 & 0.001 & 0.001 & 0.000 & 0.002 & 0.000 & 0.003 & 0.002 & 0.002 & 0.002 & 0.000 & 0.005 & 0.001 & 0.000 & 0.001 & 0.000 & 0.000 & 0.001 & 0.001 & 0.002 & 0.002 \\
\hline GAP & 0.128 & 0.391 & 0.106 & 0.009 & 0.155 & 0.073 & 0.124 & 0.187 & 0.057 & 0.201 & 0.143 & 0.038 & 0.044 & 0.068 & 0.067 & 0.126 & 0.029 & 0.119 & 0.052 & 0.081 & 0.116 \\
\hline \multirow[t]{2}{*}{ GWRP } & 0.222 & 0.519 & 0.226 & 0.030 & 0.291 & 0.095 & 0.213 & 0.313 & 0.114 & 0.303 & 0.241 & 0.125 & 0.086 & 0.100 & 0.127 & 0.256 & 0.092 & 0.204 & 0.104 & 0.212 & 0.237 \\
\hline & Gunshot & $\begin{array}{l}\text { Harmo- } \\
\text { nica }\end{array}$ & $\begin{array}{l}\mathrm{Hi}- \\
\text { hat }\end{array}$ & Keys & Knock & $\begin{array}{l}\text { Laugh- } \\
\text { ter }\end{array}$ & Meow & $\begin{array}{l}\text { Micro- } \\
\text { wave }\end{array}$ & Oboe & $\begin{array}{l}\text { Saxo- } \\
\text { phone }\end{array}$ & $\begin{array}{l}\text { Sciss- } \\
\text { ors }\end{array}$ & Shatter & $\begin{array}{l}\text { Snare } \\
\text { drum }\end{array}$ & Squeak & $\begin{array}{l}\text { Tambo- } \\
\text { urine }\end{array}$ & $\begin{array}{l}\text { Tear- } \\
\text { ing }\end{array}$ & $\begin{array}{l}\text { Tele- } \\
\text { phone }\end{array}$ & Trumpet & t Violin & $\begin{array}{l}\text { Writ- } \\
\text { ing }\end{array}$ & Avg. \\
\hline GMP & 0.001 & 0.002 & 0.001 & 0.002 & 0.001 & 0.001 & 0.000 & 0.000 & 0.001 & 0.001 & 0.000 & 0.000 & 0.002 & 0.000 & 0.001 & 0.001 & 0.001 & 0.002 & 0.003 & 0.001 & 0.001 \\
\hline GAP & 0.139 & 0.264 & 0.212 & 0.139 & 0.074 & 0.135 & 0.085 & 0.055 & 0.077 & 0.120 & 0.085 & 0.144 & 0.108 & 0.082 & 0.057 & 0.140 & 0.059 & 0.166 & 0.074 & 0.130 & 0.114 \\
\hline GWRP & 0.283 & 0.379 & 0.497 & 0.311 & 0.190 & 0.249 & 0.185 & 0.085 & 0.140 & 0.257 & 0.213 & 0.272 & 0.196 & 0.108 & 0.327 & 0.237 & 0.138 & 0.313 & 0.222 & 0.215 & 0.218 \\
\hline
\end{tabular}

TABLE IX

F1-SCORE, AUC AND MAP OF TIME-FREQUENCY SEGMENTATION AT DIFFERENT SNRS.

\begin{tabular}{|c|c|c|c|c|c|c|c|c|c|}
\hline \multirow[b]{2}{*}{ Algorithms } & \multicolumn{3}{|c|}{$20 \mathrm{~dB}$} & \multicolumn{3}{|c|}{$10 \mathrm{~dB}$} & \multicolumn{3}{|c|}{$\mathbf{0 ~ d B}$} \\
\hline & F1 & AUC & $\mathrm{mAP}$ & F1 & AUC & $\mathrm{mAP}$ & F1 & AUC & mAP \\
\hline GMP & 0.001 & 0.347 & 0.008 & 0.001 & 0.345 & 0.007 & 0.001 & 0.362 & 0.005 \\
\hline GAP & 0.215 & 0.889 & 0.230 & 0.168 & 0.880 & 0.187 & 0.114 & 0.861 & 0.143 \\
\hline GWRP & 0.324 & 0.849 & 0.268 & 0.280 & 0.845 & 0.227 & 0.218 & 0.836 & 0.175 \\
\hline
\end{tabular}

closer to the ground truth than obtained using GMP and GAP. Compared with event-wise SED, frame-wise SED does not depend on post-processing.

\section{G. Event-wise sound event detection}

Although frame-wise SED does not depend on postprocessing so is a more objective criterion, it makes more sense to have event-wise predictions. The event-wise predictions are obtained from frame-wise predictions following Section IV-C. Table VI shows that the GWRP achieves the best F1-score of 0.254 in event-wise SED. Although GMP seems to achieve the lowest ER of 1.00, GMP deletes all the events and has a deletion error of 1.00 and an insertion of 0 . On the other hand, GWRP has the lowest deletion error of 0.66 and has an insertion error of 1.45. The F1-scores drop from 0.254 to 0.227 to 0.167 under SNRs of $20 \mathrm{~dB}, 10 \mathrm{~dB}$ and $0 \mathrm{~dB}$. Table VII shows the the F1-score of event-wise SED of all sound classes. Some sound classes such as "barks", "harmonica" have higher detection F1-score. GWRP achieves the best averaged F1-score of 0.167 .
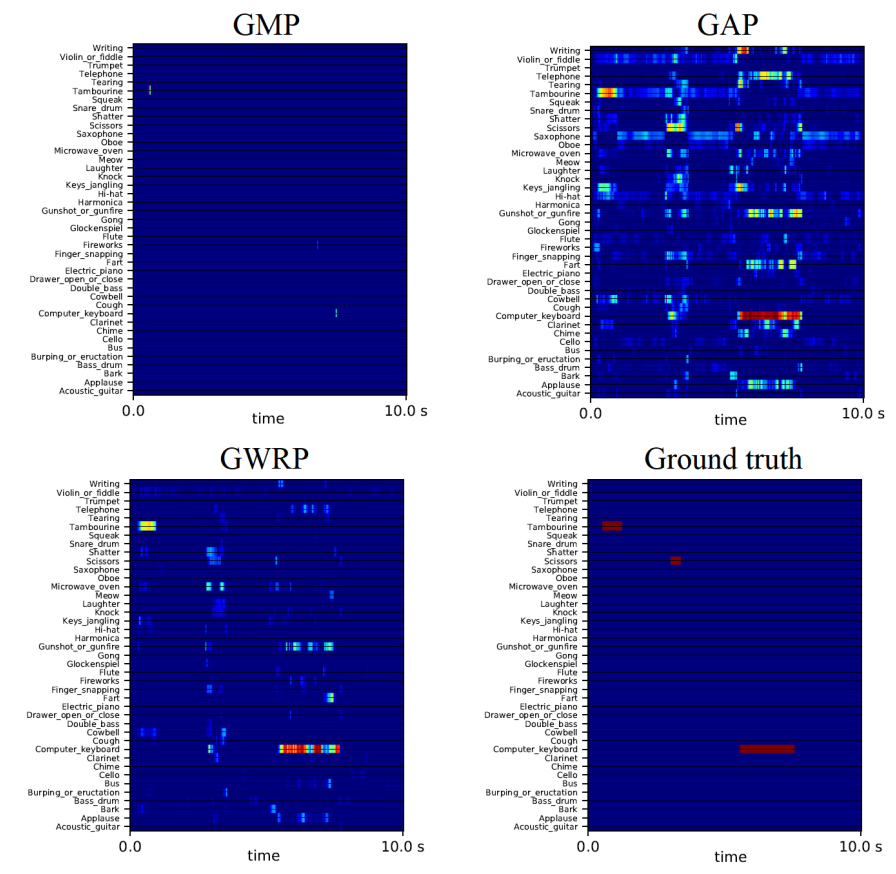

Fig. 8. Frame-wise predictions using GMP, GAP, GWRP with SNR at $0 \mathrm{~dB}$. The ground truth annotation is shown in the bottom right.

\section{H. Time-frequency segmentation}

Table VIII shows the T-F segmentation results of all sound classes under $0 \mathrm{~dB}$. As the T-F segmentation can not be obtained by previous works including the fully connected neural network [55], the CNN trained on weakly labelled data 
[37], the FrameCNN [34] and the attention model [39], we only report the T-F segmentation results with our proposed methods. GWRP achieves the best F1-score of 0.218 on average. Table IX shows the T-F segmentation results under different SNRs. Table IX shows that GWRP achieves the best F1-score, AUC and mAP of 0.324, 0.849 and 0.268 under 20 dB SNR, respectively. GMP underestimates the T-F segmentation masks and performs the worst in T-F segmentation. GAP overestimates the T-F segmentation masks and performs worse than GWRP in F1-score. The T-F segmentation masks learned by GWRP (Fig. 7(e)) looks closer to the IRM than the T-F segmentation masks learned by using GMP and GAP.

\section{CONCLUSION}

This paper proposes a time-frequency (T-F) segmentation, sound event detection and separation framework trained on weakly labelled data. In training, a segmentation mapping and a classification mapping are trained jointly using the weakly labelled data. In T-F segmentation, we use the trained segmentation mapping to calculate the T-F segmentation masks. Detected sound events can then be obtained from the T-F segmentation masks. As a byproduct, separated waveforms of sound events can be obtained from the T-F segmentation masks. Experiments show that the global weighted rank pooling (GWRP) outperforms the global max pooling, the global average pooling and previously proposed systems in both of T-F segmentation and sound event detection. The limitation of this approach is that the T-F segmentation masks are not perfectly matching the ideal ratio mask (IRM) of the sound events. In future, we will improve the T-F segmentation masks to match the IRM for event separation.

\section{ACKNOWLEDGMENT}

This research was supported by EPSRC grant EP/N014111/1 "Making Sense of Sounds" and a Research Scholarship from the China Scholarship Council (CSC) No. 201406150082. Iwona Sobieraj is sponsored by the European Union's H2020 Framework Programme (H2020-MSCA-ITN2014) under grant agreement No. 642685 MacSeNet. The authors thank Dominic Ward for helping to improve the paper in the early stage. The authors thank all anonymous reviewers for their effort and suggestions to improve this paper.

\section{REFERENCES}

[1] J. Saraswathy, M. Hariharan, S. Yaacob, and W. Khairunizam. Automatic classification of infant cry: A review. In Proceedings of the International Conference on Biomedical Engineering (ICoBE), pages 543-548, 2012.

[2] A. Harma, M. F. McKinney, and J. Skowronek. Automatic surveillance of the acoustic activity in our living environment. In Proceedins of the IEEE International Conference on Multimedia and Expo (ICME), pages 634-637, 2005.

[3] D. P. W. Ellis. Detecting alarm sounds. In Proceedings of the Consistent \& Reliable Acoustic Cues for Sound Analysis Workshop (CRAC '01), pages 59-62, 2001.

[4] G. Valenzise, L. Gerosa, M. Tagliasacchi, F. Antonacci, and A. Sarti. Scream and gunshot detection and localization for audio-surveillance systems. In Proceedings of the IEEE Conference on Advanced Video and Signal Based Surveillance (AVSS), pages 21-26, 2007.

[5] Z. Xu, Y. Yang, and A. G. Hauptmann. A discriminative CNN video representation for event detection. In Proceedings of the IEEE Conference on Computer Vision and Pattern Recognition (CVPR), pages 1798-1807, 2015.
[6] R. Girshick, J. Donahue, T. Darrell, and J. Malik. Rich feature hierarchies for accurate object detection and semantic segmentation. In Proceedings of the IEEE Conference on Computer Vision and Pattern Recognition (CVPR), pages 580-587, 2014.

[7] A. Borji, M. Cheng, H. Jiang, and J. Li. Salient object detection: A benchmark. IEEE Transactions on Image Processing, 24(12):5706$5722,2015$.

[8] Sami Abu-El-Haija, Nisarg Kothari, Joonseok Lee, Paul Natsev, George Toderici, Balakrishnan Varadarajan, and Sudheendra Vijayanarasimhan. YouTube-8M: A large-scale video classification benchmark. arXiv preprint arXiv:1609.08675, 2016.

[9] A. Narayanan and D. Wang. Ideal ratio mask estimation using deep neural networks for robust speech recognition. In Proceedings of the IEEE International Conference on Acoustics, Speech and Signal Processing (ICASSP), pages 7092-7096, 2013.

[10] D. Stowell, D. Giannoulis, E. Benetos, M. Lagrange, and M. D. Plumbley. Detection and classification of acoustic scenes and events. IEEE Transactions on Multimedia, 17(10):1733-1746, 2015.

[11] G. Parascandolo, H. Huttunen, and T. Virtanen. Recurrent neural networks for polyphonic sound event detection in real life recordings. In Proceedings of the IEEE International Conference on Acoustics, Speech and Signal Processing (ICASSP), pages 6440-6444, 2016.

[12] A. Mesaros, T. Heittola, and T. Virtanen. TUT database for acoustic scene classification and sound event detection. In Proceedings of the 24th European Signal Processing Conference (EUSIPCO), pages 11281132, 2016.

[13] D. Giannoulis, E. Benetos, D. Stowell, M. Rossignol, M. Lagrange, and M. D. Plumbley. Detection and classification of acoustic scenes and events: an IEEE AASP challenge. In Proceedings of the IEEE Workshop on Applications of Signal Processing to Audio and Acoustics (WASPAA), 2013.

[14] A. Kumar and B. Raj. Audio event detection using weakly labeled data. In Proceedings of the 2016 ACM on Multimedia Conference, pages 1038-1047, 2016

[15] S. Adavanne and T. Virtanen. Sound event detection using weakly labeled dataset with stacked convolutional and recurrent neural network. Technical report, DCASE2017 Challenge, September 2017.

[16] Qiuqiang Kong, Yong Xu, Wenwu Wang, and Mark D Plumbley. A joint separation-classification model for sound event detection of weakly labelled data. In Proceedings of the IEEE International Conference on Acoustics, Speech and Signal Processing (ICASSP), pages 321-325, 2017.

[17] O. Maron and T. Lozano-Pérez. A framework for multiple-instance learning. In Proceedings of the Advances in Neural Information Processing Systems (NIPS), volume 10, pages 570-576, 1998.

[18] Thomas G Dietterich, Richard H Lathrop, and Tomás Lozano-Pérez. Solving the multiple instance problem with axis-parallel rectangles. Artificial intelligence, 89(1-2):31-71, 1997.

[19] Z. Zhou and M. Zhang. Neural networks for multi-instance learning. In Proceedings of the International Conference on Intelligent Information Technology (ICIIT), pages 455-459, 2002.

[20] S. Andrews, I. Tsochantaridis, and T. Hofmann. Support vector machines for multiple-instance learning. In Proceedings of the Advances in Neural Information Processing Systems (NIPS), volume 15, pages 577-584, 2003.

[21] P. Siva, C. Russell, and T. Xiang. In defence of negative mining for annotating weakly labelled data. In Proceedings of the European Conference on Computer Vision (ECCV), pages 594-608, 2012.

[22] K. Tang, R. Sukthankar, J. Yagnik, and L. Fei-Fei. Discriminative segment annotation in weakly labeled video. In Proceedings of the IEEE Conference on Computer Vision and Pattern Recognition (CVPR), pages 2483-2490, 2013.

[23] A. Kolesnikov and C. H. Lampert. Seed, expand and constrain: Three principles for weakly-supervised image segmentation. In Proceedings of the European Conference on Computer Vision (ECCV), pages 695-711, 2016.

[24] A. Krizhevsky, I. Sutskever, and G. E. Hinton. ImageNet classification with deep convolutional neural networks. In Proceedings of the Advances in Neural Information Processing Systems (NIPS), volume 25, pages 1097-1105, 2012.

[25] J. Long, E. Shelhamer, and T. Darrell. Fully convolutional networks for semantic segmentation. In Proceedings of the IEEE Conference on Computer Vision and Pattern Recognition (CVPR), pages 3431-3440, 2015.

[26] G. E. Dahl, D. Yu, L. Deng, and A. Acero. Context-dependent pre-trained deep neural networks for large-vocabulary speech recogni- 
tion. IEEE Transactions on Audio, Speech, and Language Processing, 20(1):30-42, 2012.

[27] O. Abdel-Hamid, A. Mohamed, H. Jiang, L. Deng, G. Penn, and D Yu. Convolutional neural networks for speech recognition. IEEE/ACM Transactions on Audio, Speech, and Language Processing, 22(10):1533$1545,2014$.

[28] K. Choi, G. Fazekas, and M. Sandler. Automatic tagging using deep convolutional neural networks. In Proceedings of the 17th International Conference on Music Information Retrieval (ISMIR), pages 805-811, 2016.

[29] P. Foster, S. Sigtia, S. Krstulovic, J. Barker, and M. D. Plumbley. CHiME-home: A dataset for sound source recognition in a domestic environment. In IEEE Workshop on Applications of Signal Processing to Audio and Acoustics (WASPAA), 2015.

[30] M. D. Zeiler and R. Fergus. Visualizing and understanding convolutional networks. In Proceedings of the European Conference on Computer Vision (ECCV), pages 818-833, 2014.

[31] M. Lin, Q. Chen, and S. Yan. Network in network. In Proceedings of the International Conference on Learning Representations (ICLR), 2014.

[32] Q. Kong, Y. Xu, W. Wang, and M. D Plumbley. Audio set classification with attention model: A probabilistic perspective. In Proceedings of the International Conference on Acoustics, Speech and Signal Processing (ICASSP), pages 316-320, 2017.

[33] Brian McFee, Justin Salamon, and Juan Pablo Bello. Adaptive pooling operators for weakly labeled sound event detection. arXiv preprint arXiv:1804.10070, 2018.

[34] S. Chou, J. Jang, and Y. Yang. FrameCNN: A weakly-supervised learning framework for frame-wise acoustic event detection and classification. Technical report, DCASE2017 Challenge, September 2017.

[35] Ting-Wei Su, Jen-Yu Liu, and Yi-Hsuan Yang. Weakly-supervised audio event detection using event-specific gaussian filters and fully convolutional networks. In Proceedings of the IEEE International Conference on Acoustics, Speech and Signal Processing (ICASSP), pages 791-795, 2017.

[36] Shao-Yen Tseng, Juncheng Li, Yun Wang, Joseph Szurley, Florian Metze, and Samarjit Das. Multiple instance deep learning for weakly supervised audio event detection. arXiv preprint arXiv:1712.09673, 2017.

[37] Anurag Kumar and Bhiksha Raj. Deep CNN framework for audio event recognition using weakly labeled web data. arXiv preprint arXiv:1707.02530, 2017.

[38] Donmoon Lee, Subin Lee, Yoonchang Han, and Kyogu Lee. Ensemble of convolutional neural networks for weakly-supervised sound event detection using multiple scale input. In Proceedings of the Detection and Classification of Acoustic Scenes and Events (DCASE) Workshop, pages 74-79, 2017.

[39] Y. Xu, Q. Kong, W. Wang, and M. D. Plumbley. Large-scale weakly supervised audio classification using gated convolutional neural network. Proceedings of the IEEE International Conference on Acoustics, Speech and Signal Processing (ICASSP), pages 121-125, 2017.

[40] S. A. Raki, S. Makino, H. Sawada, and R. Mukai. Reducing musical noise by a fine-shift overlap-add method applied to source separation using a time-frequency mask. In Proceedings of the IEEE International Conference on Acoustics, Speech, and Signal Processing (ICASSP), volume 3, pages 81-84, 2005.

[41] S. Hershey, S. Chaudhuri, D. P. W. Ellis, J. F. Gemmeke, A. Jansen, R. C. Moore, M. Plakal, D. Platt, R. A. Saurous, B. Seybold, et al. CNN architectures for large-scale audio classification. In Proceedings of the IEEE International Conference on Acoustics, Speech and Signal Processing (ICASSP), pages 131-135, 2017.

[42] M. H. Radfar and R. M. Dansereau. Single-channel speech separation using soft mask filtering. IEEE Transactions on Audio, Speech, and Language Processing, 15(8):2299-2310, 2007.

[43] S. Ioffe and C. Szegedy. Batch normalization: Accelerating deep network training by reducing internal covariate shift. In Proceedings of the 32nd International Conference on Machine Learning (ICML), pages 448-456, 2015.

[44] V. Nair and G. E. Hinton. Rectified linear units improve restricted Boltzmann machines. In Proceedings of the 27th International Conference on Machine Learning (ICML), pages 807-814, 2010.

[45] B. Zhou, A. Khosla, A. Lapedriza, A. Oliva, and A. Torralba. Learning deep features for discriminative localization. In Proceedings of the IEEE Conference on Computer Vision and Pattern Recognition (CVPR), pages 2921-2929, 2016.

[46] K. Simonyan, A. Vedaldi, and A. Zisserman. Deep inside convolutional networks: Visualising image classification models and saliency maps. In
Proceedings of the International Conference on Learning Representations (ICLR), 2014.

[47] D. Pathak, P. Krahenbuhl, and T. Darrell. Constrained convolutional neural networks for weakly supervised segmentation. In Proceedings of the IEEE International Conference on Computer Vision (ICCV), pages 1796-1804, 2015.

[48] Jaume Amores. Multiple instance classification: Review, taxonomy and comparative study. Artificial Intelligence, 201:81-105, 2013.

[49] Annamaria Mesaros, Toni Heittola, and Tuomas Virtanen. A multidevice dataset for urban acoustic scene classification. arXiv preprint arXiv:1807.09840, 2018.

[50] Eduardo Fonseca, Manoj Plakal, Frederic Font, Daniel P. W. Ellis, Xavier Favory, Jordi Pons, and Xavier Serra. General-purpose tagging of freesound audio with audioset labels: Task description, dataset, and baseline. arXiv preprint arXiv:1807.09902, 2018.

[51] A. Mesaros, T. Heittola, and T. Virtanen. Metrics for polyphonic sound event detection. Applied Sciences, 6(6):162, 2016.

[52] J. A. Hanley and B. J. McNeil. The meaning and use of the area under a receiver operating characteristic (ROC) curve. Radiology, 143(1):29-36, 1982.

[53] A. Mesaros, T. Heittola, A. Diment, B. Elizalde, A. Shah, E. Vincent, B. Raj, and T. Virtanen. DCASE2017 challenge setup: Tasks, datasets and baseline system. In Proceedings of the Detection and Classification of Acoustic Scenes and Events (DCASE) Workshop, pages 85-92, 2017.

[54] Qiuqiang Kong, Turab Iqbal, Yong Xu, Wenwu Wang, and Mark D Plumbley. DCASE 2018 Challenge baseline with convolutional neural networks. arXiv preprint arXiv:1808.00773, 2018.

[55] Q. Kong, I. Sobieraj, W. Wang, and M. D. Plumbley. Deep neural network baseline for DCASE Challenge 2016. Proceedings of the Detection and Classification of Acoustic Scenes and Events (DCASE) Workshop, 2016.

[56] Karen Simonyan and Andrew Zisserman. Very deep convolutional networks for large-scale image recognition. In Proceedings of the International Conference on Learning Representations (ICLR), 2014.

[57] D. Kingma and J. Ba. Adam: A method for stochastic optimization. In Proceedings of the International Conference on Learning Representations (ICLR), 2015. 